\title{
Research Status of Brackish Water Desalination Technology
}

\author{
Qiaonan Yang ${ }^{1,2, \#}$, Can $\mathrm{Hu}^{1,2, \#}$, Jie $\mathrm{Li}^{1,2}{ }^{1,}$ Xiaokang $\mathrm{Yi}^{1,2,{ }^{*}}$, Jie Zhang ${ }^{1,2}$, Zhilin Sun ${ }^{3}$ \\ ${ }^{1}$ School of College of Mechanical and Electrical Engineering Tarim University, Alar 843300, China \\ ${ }^{2}$ School of Key Laboratory of Modern Agricultural Engineering Tarim University, Xinjiang Uygur Autonomous Region, Alar 843300 , \\ China \\ ${ }^{3}$ Ocean College, Zhejiang University, Zhoushan 310058, China \\ \#These authors contributed equally to this work.
}

\begin{abstract}
The serious salinization of farmland soil and the shortage of water resources in arid areas are becoming more and more serious; In order to solve the problem of shortage of fresh water resources, find a resource that can be converted into fresh water; Brackish water is derived from farmland saline alkali land. Brackish water desalination technology has increased interest in scientists' research. However, brackish water can be used as an effective substitute for freshwater conversion because of its low salt concentration and can also be widely used in many water deficient countries. However, partial or total desalting of brackish water is essential to meet the water quality requirements of production and life. The selection of appropriate water resources desalination technology needs to understand the operation methods and technical means of the existing brackish water desalination process. Proper combination of desalination technologies can improve the efficiency of brackish water desalination. This review provides the latest advances in data, materials and categories that can help select and design requirements for specific applications.
\end{abstract}

\section{Introduction}

About $75 \%$ of the earth's surface is covered by water, but only $3 \%$ is fresh water[1,2]. According to reports, fresh water is stored in glaciers, of which only $5 \%$ is easily available[3]. At present, $40 \%$ of the world's people live in arid areas or islands lacking fresh water[4]. In addition, the increase of arid areas in the world, the reduction of the adaptability of water resources to climate change, and the over development of brackish water desalination technology. The application of brackish water desalination technology affects the development of environment and economy[5]. In recent years, it is one of the major challenges faced by capitalists in the new century to bring water into the stock market. It is reported that by 2030, three fifths of the world's people will face the problem of shortage of fresh water resources[6]. In view of this problem, the state will formulate friendly policies to solve the major problem of obtaining water resources in arid areas, and ensure the basic guarantee of industrial and domestic water. Fresh water resources are very important for the economic and social development of a country and region. With the growth of the world population and the rapid economic development, the shortage of fresh water resources is becoming more and more serious. Therefore, the use of brackish water desalination technology has become a new research direction for scientists. In response to the increasing demand for fresh water resources, the key solution to avoid the shortage of fresh water supply is to develop new water resources. Desalination of brackish water is a substitute for fresh water because it is low and rich in salinity in areas facing water shortage.

At present, brackish water, as raw material for freshwater production, accounts for $21 \%$ of global raw materials. The development of energy-saving and cost-effective seawater desalination process helps to further expand the use of brackish water. At present, desalination of saline alkali water is the most useful method to solve the shortage of water resources. The combination of solar energy and membrane separation technology has been rapid development in recent years, more and more people like membrane desalination method because of simple operation, small footprint, low energy consumption and the continuous improvement of membrane material performance[7]. Membrane desalination technology mainly includes electrodialysis, reverse osmosis, membrane distillation, nanofiltration and forward osmosis. Among them, a most widely used membrane technology mainly includes electrodialysis, nanofiltration and reverse osmosis. Reverse osmosis technology has become the most advanced technology of brackish water desalination technology. However, in low brackish water desalination, RO energy efficiency will be reduced[8,9]. Nanofiltration NF can selectively remove polyvalent ions $[10,11]$, and this technology is applied to hard water softening.

The promotion of brackish water desalination technology is relatively slow. At present, membrane desalination is mainly used, and there are few studies on solar energy and membrane desalination technology. Different brackish water desalination technologies have different process

\footnotetext{
* Corresponding author: 1571832388@qq.com
} 
characteristics. Single brackish water desalination technology is not suitable for all brackish water. Improper desalination technology may cause damage to equipment, waste of water resources and even pollution. At present, there is a lack of research on the process integration technology of membrane desalination under the action of solar energy. This paper summarizes the research status of this technology, and integrates the research results of different desalination technologies to provide professional and technical personnel with.

\section{Main components of brackish water}

Brackish water refers to water with alkalinity greater than hardness and contains a large amount of neutral salt with $\mathrm{PH}$ value greater than 7 . The sources of brackish water mainly include seawater and saline alkali water. Generally, the salt content is $1 \sim 25 \mathrm{~g} / \mathrm{L}$, which is between freshwater $(<1 \mathrm{~g} / \mathrm{L})$ and seawater $(1 \sim 35 \mathrm{~g} / \mathrm{L})[12,13]$. Brackish water is divided into low salt content brackish water, medium salt content brackish water and high salt content brackish water according to salt content, and the salt content is $1-5 \mathrm{~g} / \mathrm{L}, 5-10 \mathrm{~g} / \mathrm{L}$ and $>10 \mathrm{~g} / \mathrm{L}$, respectively. The main feature of brackish water is bitter taste, and it is difficult to drink directly. If brackish water is used for a long time, it will lead to gastrointestinal dysfunction and decreased body immunity. Taking the brackish water in Xinjiang, China as an example, according to table 1, the total salt content of brackish water is $6.89 \mathrm{~g} / \mathrm{l}$, and no heavy metals such as $\mathrm{Pb}, \mathrm{CD}, \mathrm{Cr}, \mathrm{Hg}$ and $\mathrm{Sn}$ are detected.

Table 1. Composition of brackish water

\begin{tabular}{ccc}
\hline $\begin{array}{c}\text { Ion Composition } \\
\text { of Brackish } \\
\text { Water }\end{array}$ & $\begin{array}{c}\text { Ion mass } \\
\text { concentration } \\
(\mathrm{mg} / \mathrm{L})\end{array}$ & $\begin{array}{c}\text { Percentage of } \\
\text { ion mass } \\
\text { concentration } \\
(\%)\end{array}$ \\
\hline $\mathrm{Na}+$ & 1674.03 & 26.32 \\
\hline $\mathrm{K}+$ & 19.47 & 0.31 \\
\hline $\mathrm{Ca} 2+$ & 434.60 & 6.83 \\
\hline $\mathrm{Mg} 2+$ & 291.80 & 4.59 \\
\hline $\mathrm{Cl}-$ & 2624.48 & 41.26 \\
\hline $\mathrm{SO} 42-$ & 1315.94 & 20.69 \\
\hline $\begin{array}{c}\text { total } \\
\text { mineralization }\end{array}$ & 6360.32 & 100 \\
\hline
\end{tabular}

\section{Development Status of Brackish Water}

\subsection{Utilization of Brackish Water in Agriculture}

The development and utilization of brackish water resources is an effective method to alleviate the shortage of fresh water in arid areas. On the whole, the global brackish water content is as high as $1.45 \times 10^{9} \mathrm{~km}^{3}$, accounting for $3 \%$ of the total water volume of the earth. After desalination, it can meet the demand for fresh water[14]. Brackish water is widely distributed in areas. Desalination of brackish water can reduce the salinization harm of brackish water to farmland soil and meet the water consumption of agricultural irrigation[15,16]. In normal farmland irrigation, salts in brackish water, such as $\mathrm{Na}^{2+}, \mathrm{Ca}^{2+}, \mathrm{Mg}^{2+}, \mathrm{SO}_{4}{ }^{2-}$, will provide fertilizer efficiency for crops to a certain extent and save planting costs [17-19].

Brackish water desalination technology has gradually entered the stage of industrial production from small-scale experimental desalination, providing a strong guarantee for drinking water and agricultural and forestry irrigation water, such as Israel, the United States, Italy, France, Austria and other countries [20]. Especially in some countries with extreme water shortage in the Middle East: Saudi Arabia, the United Arab Emirates, Israel, etc., as well as Japan, Spain and the United States in developed countries, there are more and more successful examples of brackish water desalination. The success of brackish water desalination technology has played a grass-roots guarantee role for local industrial water, domestic water, farmland irrigation water and ecological environment[21].

\subsection{Utilization of Brackish Water in Breeding Industry}

It is completely feasible to cultivate P. chinensis in salinealkali areas far from the sea area, and its benefit is much higher than that of other varieties[22]. During the whole breeding process, the daily management is the same as that of shrimp farming in coastal China. As long as the salinity of groundwater in saline-alkali land can reach more than $25 \%$ and there is good underground freshwater resources, shrimp culture in China can be carried out. In order to solve the pressure of freshwater aquaculture, worldwide use of brackish water to develop aquaculture. The United States, Israel, India and Australia have begun to use farmland brackish water desalination technology for commercial production of fresh water[23]. At present, Texas, Arizona, Arkansas, Alabama and Florida in the United States have evaluated the cultivation of inland brine shrimp, and the cultivation of Litopenaeus vannamei in Texas began in 1970. Water in Arizona was used to cultivate shrimp in ponds and kandao, and aquaculture wastewater was used to irrigate crops. Australia also carried out small-scale aquaculture production of squatfish using Weishui in southwest China.

\section{Research Progress of Brackish Water Desalination Technology}

\subsection{Membrane Desalination Technology under Solar Energy}

The water in the solar distiller is distilled by absorbing the radiant heat of the sun. The latest distillation technology is based on membrane distillation and multistage distillation. In the process of distillation, the energy required to produce 1 ton of fresh water is $30-80 \mathrm{kw}$ [24]. In 2011, winter et al. [25] came from Fraunhofer Solar Energy Research Institute. They considered winding the frame on the damaged MD module, with a surface area of $9-14 \mathrm{~m} 2$. The test results show that the salinity of feed flow and feed water has a significant effect on the boundary of damaged module. In 2012, Chang et al. [26] studied a 
solar based thin film refining and desalination framework smdds, which uses porous layer refining. The frame is composed of cold liquid indoor regulator, hot temperature storage tank, siphon and energy recovery device. The results showed that the distillate was $0.358 \mathrm{~kg} / \mathrm{d}$ on the day with radiation, and $0.152 \mathrm{~kg} / \mathrm{d}$ on the cloudy day. In 2015 , aim et al. [27] introduced a closed desalination system in a manor in Dubai. The desalination system can meet the demand of $260 \mathrm{~L} / \mathrm{d}$ high-temperature water and 18-25 L/d pure drinking water. The desalination unit adopts a pore layer with an opening area of $11.85 \mathrm{~m}^{2}$ to refine the seawater desalination cycle and horizontal plate gas collector. In 2021, Lacroix introduced an innovative thermodynamic reverse osmosis desalination device [28]. The desalination process of this new desalination device uses the heat energy of the sun to pressurize the salt water above its osmotic pressure to desalinate it.

\subsection{Electrodialysis desalination technology}

Membrane based desalination technology includes osmotic membrane to separate salt molecules from water. The most commonly used membrane separation technologies are electrodialysis and reverse osmosis. In the electrodialysis process, the salt in the solution is discharged through two electrodes through the selective membrane. This technology is suitable for the desalination of brackish water with salt concentration lower than $3 \mathrm{~g} / \mathrm{L}^{-1}$ [29]. Early electrodialysis methods were widely used in brackish water and seawater desalination, chemical industry, wastewater treatment and food industry [30]. With the development of science and technology, the performance of ion exchange membrane used in electrodialysis process and the process structure of electrodialysis device have been continuously innovated and improved, and have also been widely used in new fields such as bioengineering, medicine, flue gas desulfurization and wastewater treatment in coalbed methane, natural gas and oil manufacturing [31]. Electrodialysis technology has high operability in terms of desalting effect in certain environment and treatment of certain types of brackish water. It can meet the requirements of crops for water with different salt content. The technical cost and energy consumption cost can be reduced to a certain extent and has good flexibility.

\subsection{Membrane desalination technology}

Reverse osmosis technology is a new membrane separation technology, which is widely used in the process of brackish water desalination. In 1992, Rodgers et al. [32] developed a model to apply pressure to the membrane. They studied the effect of this pressure on the permeability of reverse osmosis membrane, indicating that negative pressure membrane pressure may increase the permeability of reverse osmosis membrane and optimize it. In 2005, Kim et al. [33] compared the research results of three-dimensional model and two-dimensional numerical value on the theoretical properties of thin films. After experimental verification, the research results showed the correlation between the theoretical model of thin films and working stability conditions. In 2019, Ali et al. Also introduced the motion model of membrane [34] and used mathematical methods to show that the high-step change of feed mass flow will indeed significantly affect the permeation effect of reverse osmosis membrane. In addition to these models, the reverse osmosis desalination technology is also studied. In 2021, cheddie et al. [35] studied the reverse osmosis process of seawater under wave pressure, and introduced the permeation model of reverse osmosis device. The experimental results show that periodic pressure application can minimize the polarization effect and improve the performance. These work show that dynamic operating conditions affect the performance of RO membrane, and the membrane behavior needs to be modeled in a dynamic way to evaluate these effects.

\section{Existing Problems and Solutions}

\subsection{Problems faced by reverse osmosis technology}

Compared with other technologies, reverse osmosis technology has relatively low energy consumption, but the demand for seawater desalination technology is increasing, which means that more and more energy is used. According to the recent and future prediction of estimated energy pollution, in 2045, the global emissions of carbon dioxide and brackish water desalination technology will reach 35 million tons per year [36]. Kuwait and other countries use part of their oil production for brackish water desalination and national power generation, which has a great impact on environmental pollution, depletion of renewable energy, shortage of water resources, more serious destruction of ozone layer and toxic compounds in groundwater [37]. Environmental protection and freshwater resource utilization technology are the common research goals of many scientists, because many scientists provide better ideas for the expansion of reverse osmosis technology.

\subsection{Continue to Increase the Promotion of Brackish Water Desalination}

After more than 20 years of development, brackish water desalination equipment has been popularized to some extent, but there are still serious drinking water problems in some economically underdeveloped areas. Therefore, the promotion of electrodialysis and reverse osmosis brackish water desalination methods and support policies need to be vigorously promoted and supported by the government.

\subsection{Rational development to ensure long-term sustainable use}

Brackish water resources are an integral part of the total water resources, which also need reasonable development to ensure long-term use. At present, the total amount of 
brackish water resources in China is 198 billion m3/a. Brackish water is the main source of drinking water in some areas, so brackish water and freshwater resources need to be planned and exploited. Therefore, in the shallow brackish water distribution area, brackish water desalination should be comprehensively promoted. The exploitation of shallow underground brackish water should be encouraged. On the one hand, it is convenient to take water. On the other hand, the exploitation of shallow underground brackish water will not lead to ground subsidence, and it is conducive to the recovery of groundwater quality and the improvement of ground soil environment. In line with the principle of "light first and then salty, shallow first and then deep," flexible and diverse forms are adopted to provide healthy drinking water resources for the vast brackish water areas.

\section{Conclusion}

Fresh water can be obtained from brackish water, saline alkali water and seawater. The most suitable technology for obtaining fresh water resources is to use brackish water with relatively low salt content. Through the combination of other technologies, the recovery rate of fresh water can be improved, so as to minimize the generation of wastewater. Solar energy technology and membrane separation technology, electrodialysis method and membrane separation technology have been widely explored for the treatment of brackish water desalination because of the low energy demand. In recent decades, the simplification of device design and operation, the improvement of membrane technology and the development of electrodialysis methods can reduce the cost of brackish water desalination and make these technologies more visible. However, the consumption of membrane and the working environment of membrane are still limited, so membrane needs to be explored in brackish water desalination technology. Understanding these technologies can make up for the deficiency of brackish water desalination technology in research.

\section{Acknowledgments}

The project support for this work includes: major science and technology projects of BINGTUAN: 2018AA003, graduate innovation project of Tarim University: TDGRI202039.

\section{References}

1. he Chinese Society of Agricultural Engineering, 2008,24(10): 243-247.

2. Q.S. Zhang, Z. Lou. Application of electrodialysis technology in desalination of amino acids, pharmaceutical intermediates, juice and high salinity wastewater . Qingdao International Desalting Conference, 2012.
3. L. Yu. The Application of Electrodialysis Technique in Food and Pharmaceutical lndustry. China Environment Management, 2010.

4. Y.C. Xiao, R.Y. Chen, X. Zheng, et al. Preparation of $\mathrm{P}-\mathrm{mSA} / \mathrm{mCS}$ bipolar membrane and its application in electrosynthesis of 2,3,5,6-tetrachlopyridine. CIESC Journal, 2010, 61(2):525-530.

5. J. Zhao, B. Feng. Study on the electrodialysis desalination of amino acid from fermentation broth. Food and Fermentation Industries. 2006,32(9):32-36.

6. O.M. Kattan, H.J. Mengers, et al. On the isolation of single acidic amino acids for biorefinery

7. applications using electrodialysis. Journal of Membrane Science, 2011. 384: 166-175.

8. R. Benjamin, S. Philippe, et al. Application of relaxation periods during electrodialysis of a casein

9. solution: Impact on anion-exchange membrane fouling. ScienceDirect, 2007. 287:41-50.

10. V. Rodgers, R. Sparks. Effect of transmembrane pressure pulsing on concentration polarization. Membr. Sci. 1992, 68, 149-168.

11. S. Kim, E.M. Hoek. Modeling concentC. Chen, Y. Jiang, Z. Ye, Y. Yang, et al. Sustainably integrating desalination with solar power to overcome future freshwater scarcity in China. Glob. Energy Interconnect.2019,2, 98-113.

12. C. Zhao, Q.Q. Gao, Y.H. Zhang, J.J. Song, F.Z. Sun. Research progress of brackish water desalination technology. Water Resources Development Research. 2021,21(08):61-65.

13. R.X. Hua, Y.Y. Gao, X.O. Feng. Experimental study on desalination of brackish water in plastic film evaporation shed. Gansu Water Resources and Hydropower Technology. 2021,57(06):16-20.

14. Y. Ibrahim, R.A. Ismail, A. Ogungbenro, et al. The sociopolitical factors impacting the adoption and proliferation of desalination: A critical review. Desalination 2021, 498, 114798.

15. A. Tappe. Investors Can Now Trade Water FuturesCNN. Available online: https:/edition.cnn.com/2020/12/07/investing/waterfutures-trading/index.html (accessed on 12 December 2020).

16. D. Zetland. The role of prices in managing water scarcity. Water Secur.2021,12, 100081.

17. K.A. Wang, .Mechanical properties of waterdesalination and wastewater treatmentmembranes. Desalination, 2017, 401:190205.

18. T. Qiu, P.A. Davies. Comparison of configurations for high-recovery inland desalination systems. Water 2012, 4, 690-706.

19. A. Drak, M. Adato. Energy recovery consideration in brackish water desalination. Desalination 2014, 339, 34-39.

20. H. Yacubowicz, J. Yacubowicz. Nanofiltration: Properties and uses. Filtr. Sep. 2005, 42, 16-21. 
21. W. Chen, M. Jin, P. Ferre, et al. Spatial distribution of soil moisture, soil salinity, and root density beneath a cotton field under mulched drip irrigation with brackish and fresh water. Field Crop. Res. 2018, 215, 207-221.

22. F. Ren, G. Yang, W. Li, et al. Yield-compatible salinity level for growing cotton (Gossypium hirsutum L.) under mulched drip irrigation using saline water. Agric. Water Manag. 2021, 250, 106859.

23. N. Ghaffour, M.M Thomas, L.A. Gary. Technical review and evaluation of the economics of water desalination: current and future challenges for better water supply sustainability. Desalination, 2013,309: 197-207.

24. A. Maurel. Brackish Water Desalination and Other Non-Conventional Processes for Water Supply. Desalination, 2006,4(5): 72-73.

25. Y.F. Song, X.F. Su, J.G. Zhou, B.W. Su, C.X. Gao. Application and research progress of brackish water desalination process with high recovery membrane method. Chemistry. 2015.78(01):79.

26. G.H. Xiao, L.J. Liu, C.W. Zhang, M.Z. Zhao. Experiment on culturing Penaeus chinensis in brackish water in saline alkali land. Hebei Fisheries. 2006(08):30-34.

27. M.S. Abujazar, S. Fatihah, A.R. Rakmi,et al. The effects of design parameters on productivity performance of a solar still for seawater desalination: A review. Desalination, 2016. 385: 178-193.

28. B. Chaouchi, A. Zrelli, S. Gabsi. Desalination of brackish water by means of a parabolic solar concentrator. Desalination, 2007. 217(1): 118-126.

29. Bundschuh, Jochen, et al. Low-cost low-enthalpy geothermal heat for freshwater production: Innovative applications using thermal desalination processes. Renewable and Sustainable Energy Reviews, 2015. 43: 196-206.

30. H. Aburideh, A. Deliou, B. Abbad, et al. An experimental study of a solar still: Application on the sea water desalination of Fouka. Procedia Engineering, 2012. 33: 475-484.

31. G.H. Xiao, L.J. Li,C.W. Zhang, et al. Experiment on Chinese shrimp culture in brackish water in salinealkali soil. Hebei Fisheries,2006(08):30+34.

32. W.L. Xu, H.Q. Wang, Y.H. Li. Distribution and aquaculture application of saline alkali water at home and abroad . China Fisheries,2021(07):50-53.

33. D. Winter, J. Koschikowski, M. Wieghaus. Desalination using membrane distillation: Experimental studies on full scale spiral wound modules. Membr. Sci. 2011, 375, 104-112.

34. H. Chang, S.G. Lyu, C.M. Tsai, et al. Experimental and simulation study of a solar thermal driven membrane distillation desalination process. Desalination 2012, 286, 400-411.

35. M. Asim, N.T. Uday Kumar, A.R. Martin. Feasibility analysis of solar combi-system for simultaneous production of pure drinking water via membrane distillation and domestic hot water for single-family villa: Pilot plant setup in Dubai. Desalination Water Treat. 2015, 57, 21674-21684.

36. Q.S. Wang, T.J. Ying,C.Y. Zhao, et al. Application of electrodialysis technology on desalination of soybean oligosaccharides solution. Transactions of tration polarization in reverse osmosis processes. Desalination 2005, 186, 111-128.

37. H. Nassrullah, S.F. Anis, R. Hashaikeh, et al. Energy for desalination: A state-of-the-art review. Desalination 2020, 491, 114569. 